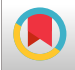

\title{
Prevalence of Undescended Testis in Tehran and the Main Risk Factors: A Cross-sectional Multicenter Study
}

\author{
Alimohammad Fakhr Yasseri ${ }^{1}$, Hossein Dialameh (iD ${ }^{1,}{ }^{*}$, Farshad Namdari ${ }^{1}, 2,{ }^{, * *}$, Ghazal Shariatpanahi \\ (iD ${ }^{1}$, Maryam Veisizadeh ${ }^{1}$, Alireza Gorji ${ }^{1}$, Alipasha Meisami ${ }^{3}$, Parvaneh Soleimani ${ }^{1}$ and Soheila \\ siroosbakht (iD ${ }^{4}$ \\ ${ }^{1}$ Tehran University of Medical Sciences, Sina Hospital, Tehran, Iran \\ ${ }^{2}$ AJA University of Medical Sciences, Tehran, Iran \\ ${ }^{3}$ Epidemiology and Preventive Medicine Department, Tehran University of Medical Sciences, Tehran, Iran \\ ${ }^{4}$ Department of Prdiatrics, AJA University of Medical Sciences, Tehran, Iran \\ "Corresponding author: Tehran University of Medical Sciences, Sina Hospital, Tehran, Iran. Email: hossein.dialameh@gmail.com \\ "Corresponding author: Tehran University of Medical Sciences, Sina Hospital, Tehran, Iran. Email: farshad.namd@yahoo.com
}

Received 2020 November 11; Revised 2021 January 10; Accepted 2021 January 11.

\begin{abstract}
Background: Undescended testis (UDT) is the most common abnormality of the male genital system. To date, no study has been carried out to determine the prevalence of UDT and its risk factors in Tehran. This study aimed to determine the frequency of cryptorchidism and its risk factors in male infants who were born at three university hospitals in Tehran.

Methods: A cross-sectional study was done on boys born at three major university hospitals (Baharlou, Yas, and Ziaeian Hospitals) in Tehran between 2016 and 2018. All of the newborns were examined on the first or second day of birth. Demographic data were collected using hospital databases after getting permission. Also, the pregnancy care card was used to collect maternal complications of pregnancy, including gestational diabetes mellitus, preeclampsia, eclampsia, fetal malignancy, preterm, and late delivery.

Results: A total of 1,350 boys were evaluated. Eleven ( $0.81 \%$ ) boys had UDT. There were statistically significant associations between UDT and maternal hypertension, presence of other congenital anomalies, infection during pregnancy, fetal presentation, and type of delivery. The average birth weight and gestational age were lower in patients with UDT than in healthy infants.

Conclusions: The prevalence of UDT in Tehran was much lower than in previously reported rates in other areas. Factor analysis showed that cryptorchidism was associated with the markers of poor fetal development.
\end{abstract}

Keywords: Cryptorchidism, Risk Factors, Prevalence, Iran

\section{Background}

Cryptorchidism is the most common abnormality of the male genital system. The definition of cryptorchidism or undescended testis (UDT) is the lack of testicles in their normal locations. The inability to touch the testicle can be due to its absence (agenesis) or lack of descent. Both agenesis and impaired antenatal blood flow (such as torsion) can be the cause of non-palpable testicles. However, anorchia refers to cases with no testicles on either side $(1,2)$.

Furthermore, many cases of UDT are underdeveloped. These abnormalities occur when the descending process of the testicles stops because the testes develop in the abdomen during embryonic development. Their descent and migration may stop anywhere along the path or deviate to an abnormal location (ectopic testis) along the descent. Besides, UDT may remain in the abdomen or could be palpable in the inguinal canal or just outside the external ring of the inguinal channel (supra-scrotal) $(1,3)$. The evaluation of non-palpable UDT usually starts with an ultrasound examination. If the testis was non-visible on ultrasound, further evaluations would be necessary. Magnetic resonance imaging (MRI) has been the imaging of choice for these cases for many years; however, nowadays, the role of MRI is decreased. Laparoscopic exploration has been the gold standard for these cases recently, which not only is accurate for finding testicles but also can simultaneously provide treatment for these patients $(1,4)$.

Moreover, there are some similar conditions, such as retractile testis, in which the testicles may often be palpable in the scrotum, but they will sometimes pull out of the scrotum if the cremasteric reflex activates (1). This disease is not classified as cryptorchidism, and its treatment is different (1). 


\section{Objectives}

To date, no study has been carried out to determine the prevalence of UDT and its risk factors in Tehran. This study aimed to determine the frequency of cryptorchidism and its risk factors in male infants who were born at three university hospitals in Tehran. So, health care officials could better plan for this disease.

\section{Methods}

A cross-sectional study was conducted on male infants who were born at three major hospitals in Tehran (Baharloo, Yas, and Ziaian University Hospitals) between 2016 and 2018. The inclusion criteria were live birth, male gender, and parental consent. The exclusion criteria were stillbirth and parents' unwillingness to participate in the study.

A form was designed to record the data. Demographic data were collected using hospital databases after getting permission. Also, the pregnancy care card was used to collect maternal risk factors and complications of pregnancy, including smoking, analgesic use, maternal hypertension, fever during the first trimester, infection during pregnancy, history of exposure to toxins and pesticides, gestational diabetes mellitus, preeclampsia, eclampsia, fetal malignancy, preterm and late delivery, and type of delivery. Fetal complications such as low birth weight and age at birth were also recorded in the forms. Sampling was performed by a non-probabilistic method and continued until the desired sample size was reached. All infants were examined on the first or second day after birth. Examinations were performed at postnatal wards at normal room temperature $\left(21\right.$ to $\left.24^{\circ} \mathrm{C}\right)$ in the supine position. The position of the testicle after lowering the testicle to the farthest point along the natural descent path without high tensile strength was recorded and classified as normal (normal scrotal testicles or retractile) or UDT (suprascrotal testes, non-palpable, or inguinal).

Babies who were born before 37 weeks of gestation were considered preterm. Gestational age was estimated at 18 to 20 weeks using ultrasound results. In other cases, the time of the last menstrual period and the Dubowitz score (5) after birth were used to determine gestational age. Spontaneous abortion, preeclampsia, fetal hypoxia, and multiple gestations were considered abnormal pregnancies. Any types of congenital malformations, which were found in the physical examination, were included. Low birth weight was defined as a weight less than 2,500 grams. The national birth weight chart by gender was used to calculate the small for gestational age (SGA), and a weight of less than 0.24 of the predicted size (SGA) was considered. Further information, including job conditions, smoking habits, and opioid use, was gathered by questionnaires.

The collected data were analyzed by SPSS software, version 21. The chi-square and Fisher's extract tests were used to examine qualitative data, and a t-test was used to examine quantitative data. The significance level was considered less than 0.05 .

\section{Results}

Among 1,350 male infants who were evaluated, the prevalence of UDT was $0.81 \%$ (11 patients). Average maternal and paternal ages were $26.09 \pm 3.88$ and $29.27 \pm 3.79$ in the UDT group, respectively. Average neonatal weight and average gestational age were $2,454.54 \pm 760.74$ grams and $37.63 \pm 1.74$ weeks in the UDT group, respectively. Besides, $7.4 \%$ of mothers in the UDT group had gestational hypertension, and there was a significant relationship between hypertension and UDT $(\mathrm{P}<0.05)$. Also, 5.3\% of mothers in the UDT group had gestational diabetes, but there was no significant relationship between gestational diabetes and UDT. There was a significant relationship between congenital anomalies and UDT, so that $33.3 \%$ of infants born with cryptorchidism had congenital malformation $(\mathrm{P}<0.01)$. In addition, 5.6\% of mothers in the UDT group experienced infections during pregnancy, and there was a significant relationship between pregnancy infections and this disease $(\mathrm{P}<0.05)$. In the UDT group, $1.9 \%$ of mothers had a history of fever in the first trimester of pregnancy, but there was no significant relationship between fever and UDT (P $>0.05$ ).

The fetal presentation was breech in $0.7 \%$ of UDT patients. There was a significant relationship between fetal presentation and cryptorchidism $(\mathrm{P}<0.05)$. Besides, 3\% of mothers of infants with cryptorchidism used analgesics during pregnancy, but there was no significant relationship between analgesics use and UDT. None of the mothers of children with cryptorchidism had a history of steroid use or exposure to pesticides during pregnancy. Furthermore, there was no significant relationship between maternal blood type or Rh group and UDT. There was also no relationship between treatment of infertility and cryptorchidism. However, the relationship between the type of delivery and UDT was significant $(\mathrm{P}<0.05)$.

To simultaneously investigate the effect of various factors on the variables that showed a significant relationship in univariate analysis or chi-square test, they were simultaneously entered into a logistic model, and their combined effect was evaluated. Gestational hypertension had a great effect on the main model and showed a significant direct relationship with cryptorchidism, so that the odds ratio was about 12. Congenital anomalies had the greatest effect 
on the regression model, and its odds ratio was 165 . Infection during malignancy was the second factor (odds ratio= 15). The other variables were not effective in the regression model and were excluded from the final model.

\section{Discussion}

In recent decades, the incidence of UDT has increased in both the United Kingdom $(6,7)$ and the United States of America (8). However, our study and some other studies have reported a lower prevalence of cryptorchidism. These contradictions may indicate geographical or temporal differences in the incidence of cryptorchidism or differences in treatment patterns, diagnostic criteria, or study designs. The prevalence of cryptorchidism at birth was reported between 2 and 3\% in previous studies, and its prevalence was between 0.8 to one percent in one-year-old children (4), but the results of our study indicated a $0.8 \%$ prevalence of cryptorchidism at birth. Although there are some reports with higher rates of UDT in our country (2), there are many studies with a similar prevalence. For instance, a population-based study on 390,344 male infants in Washington showed that the prevalence of cryptorchidism was around $0.5 \%$, which is even lower than the prevalence in our study (8).

Although the main mechanism of testicular descent is not well understood, it is thought that complex reactions between hormonal, mechanical, or anatomical factors are involved. The current model for testicular descent consists of two independent stages: the trans-abdominal stage (in early pregnancy) and the inguinoscrotal stage (in the third trimester). Since most UDTs ( 81 to $83 \%$ ) are found in the groin, the abnormality in the inguinoscrotal stage could be more prevalent, which depends on the secretion of fetal testosterone produced by human chorionic gonadotropin. Estradiol may also play a role in the development of UDT by inhibiting testosterone secretion or exerting a direct estrogenic effect on embryogenic paratesticular structures, thereby interfering with their normal development $(9,10)$.

Prematurity is a strong risk factor for cryptorchidism (9), but this association does not seem to be independent of birth weight among boys who remain cryptorchid for at least one year after birth (10). The results of our study also showed that increasing gestational age is a protective factor against the occurrence of cryptorchidism. Considering that testicular descent into the scrotum occurs in the late stages of pregnancy, it can be stated that the occurrence of cryptorchidism in preterm infants indicates a delay in development due to the short length of pregnancy. On the other hand, a strong association was reported between cryptorchidism and low birth weight in the literature (9). Similarly, this association was seen in our study population.
In the present study, mothers were not exposed to toxins and pesticides, and therefore, the possibility of investigating the role of these factors in the development of UDT could not be assessed. However, some studies have reported an increased risk of cryptorchidism in infants with mothers working in farms and greenhouses Biggs et al. conducted a study in Spain and showed that a positive association between the rate of orchiopexy and the use of insecticides (11). Some insecticides have been shown to have anti-androgenic effects and interfere with the development of the reproductive system in laboratory animals (12).

We did not find any association between cryptorchidism and smoking in mothers; however, there was a weak positive association between smoking during pregnancy and the incidence of cryptorchidism in a recent meta-analysis (3). In our study, five out of 11 infants with cryptorchidism had a breech presentation and undergone cesarean sections. Some researchers mentioned that the breech presentation is ineffective in $\operatorname{UDT}(13,14)$; however, other studies, like ours, have considered this factor (6, 10). This finding does not appear to be due to circulatory disturbances or mechanical damage to the testicles as a result of increased breech delivery duration because almost all of these infants are born by cesarean sections.

In our study, all infants with UDT had other congenital anomalies. Previous studies have reported a strong association between cryptorchidism and an increase in the prevalence of congenital anomalies (13-16). Cryptorchidism is a common component of many congenital syndromes such as Prader-Willi's and Prune-belly syndromes. This strong association between genitourinary tract and gastrointestinal malformations and cryptorchidism can be due to decreased abdominal pressure or obstruction resulting in impaired testicular descent (17).

There is little evidence of an association between cryptorchidism and maternal diseases such as diabetes, preeclampsia, or hypertension during pregnancy $(10,13)$. However, our study showed a significant association between hypertension and UDT, while no association was observed between gestational diabetes and UDT. Hypertensive disorders, diabetes, and kidney disease in mothers are known risk factors for fetal growth retardation (18) and may therefore be involved in UDT.

The main limitation of this study was the evaluation of cryptorchidism at birth only. In approximately $71 \%$ of infants with UDT at birth, spontaneous descending of the testis occurs within a year without any medical intervention (9). However, our results are consistent with the results of population-based studies that selected boys undergoing orchiopexy as the target group $(10,13,14)$. Another limitation of our study is that we probably did not have complete case ascertainment, and this may have in- 
fluenced the observed prevalence. A study of the completeness of birth defects in hospital data in the USA found that $36 \%$ of infants with UDT had not been reported (19). It is unclear whether our prevalence is accidental or is related to maternal risk factors or pregnancy complications. Another limitation was the small sample size in the UDT group, which interfered with our analysis. There was no control of confounding factors in the analysis, as well. It would be better to use correlation tests (two-tailed Pearson's correlation coefficient analyses). However, our findings are largely consistent with other similar studies with probably more complete evidence. Larger national population-based studies could help have a better estimation of this prevalence in Iran.

\subsection{Conclusions}

Our study showed that the prevalence of UDT in Tehran is much lower than that in previous studies. Cryptorchidism seems to be associated with markers of poor embryonic development. Low gestational age, low birth weight, breech presentation, and consequently, a cesarean section for birth and history of maternal infection during pregnancy were more common in infants with cryptorchidism than in other infants.

\section{Acknowledgments}

We would like to express our special thanks to our professor, Shahram Gooran, who gave us the golden opportunity to do this wonderful research; however, we, unfortunately, lost him before the end of this project. He was one of our best professors, not only in the field of urology and kidney transplant but also in humanity and faithfulness.

\section{Footnotes}

Authors' Contribution: Study concept and design: H.D., and A.Y.; Analysis and interpretation of data: A.M.; Drafting of the manuscript: G.S.; Critical revision of the manuscript for important intellectual content: F.N, P.S., and M.V.; Statistical analysis: A.G. and A.M.

Conflict of Interests: There is no conflict of interest. Funding/Support: None.

\section{References}

1. Partin AW, Peters CA, Kavoussi LR, Dmochowski RR, Wein AJ. CampbellWalsh-Wein Urology Twelfth Edition Review E-Book. 3rd ed. Elsevier Health Sciences; 2020.

2. Amooei A, Kargar S. Prevalence and pathological features of cryptorchidism among Iranian children in Yazd province, central Iran. Int JPediatr.2018;6(5):7615-21. doi: 10.22038/ijp.2018.29435.2576.
3. Yu C, Wei Y, Tang X, Liu B, Shen L, Long C, et al. Maternal smoking during pregnancy and risk of cryptorchidism: a systematic review and meta-analysis. Eur J Pediatr. 2019;178(3):287-97. doi: 10.1007/s00431018-3293-9. [PubMed: 30465272].

4. Desire AM, Buhendwa C, Cesaire TMA, Prisca KIS, Levi LN, David $\mathrm{NB}$, et al. Epidemiology, diagnosis and therapeutic approaches of cryptorchidism at the panzi general hospital, dr congo: A 5-year retrospective study. Ethiop J Health Sci. 2020;30(1):107-14. doi: 10.4314/ejhs.v30i1.14. [PubMed: 32116439]. [PubMed Central: PMC7036455].

5. Vik T, Vatten L, Markestad T, Jacobsen G, Bakketeig LS. Dubowitz assessment of gestational age and agreement with prenatal methods. Am J Perinatol.1997;14(6):369-73. doi:10.1055/s-2007-994163. [PubMed: 9217961].

6. Swerdlow AJ, Wood KH, Smith PG. A case-control study of the aetiology of cryptorchidism. J Epidemiol Community Health. 1983;37(3):23844. doi: 10.1136/jech.37.3.238. [PubMed: 6137506]. [PubMed Central: PMC1052300].

7. Chilvers C, Forman D, Pike MC, Fogelman K, Wadsworth MEJ. Apparent doubling of frequency of undescended testis in England and Wales in 1962-81. Lancet. 1984;324(8398):330-2. doi: 10.1016/s01406736(84)92697-7.

8. Schottenfeld D, Warshauer ME, Sherlock S, Zauber AG, Leder M, Payne R. The epidemiology of testicular cancer in young adults. Am J Epidemiol.1980;112(2):232-46. doi: 10.1093/oxfordjournals.aje.a112989. [PubMed: 6106385].

9. Berkowitz GS, Lapinski RH, Dolgin SE, Gazella JG, Bodian CA, Holz man IR. Prevalence and natural history of cryptorchidism. Pediatrics. 1993;92(1):44-9. [PubMed: 8100060].

10. Jones ME, Swerdlow AJ, Griffith M, Goldacre MJ. Prenatal risk factors for cryptorchidism: a record linkage study. Paediatr Perinat Epidemiol. 1998;12(4):383-96. doi: 10.1046/j.1365-3016.1998.00144.x. [PubMed: 9805712].

11. Biggs ML, Baer A, Critchlow CW. Maternal, delivery, and perinatal characteristics associated with cryptorchidism: a populationbased case-control study among births in Washington State. Epidemiology. 2002;13(2):197-204. doi: 10.1097/00001648-200203000-00015 [PubMed: 11880761].

12. Cognez N, Warembourg C, Zaros C, Metten MA, Bouvier G, Garlantezec $\mathrm{R}$, et al. Residential sources of pesticide exposure during pregnancy and the risks of hypospadias and cryptorchidism: the French ELFE birth cohort. Occup Environ Med. 2019;76(9):672-9. doi: 10.1136/oemed2019-105801. [PubMed: 31413190].

13. Hjertkvist M, Damber JE, Bergh A. Cryptorchidism: A registry based study in Sweden on some factors of possible aetiological importance. J Epidemiol Community Health. 1989;43(4):324-9. doi: 10.1136/jech.43.4.324. [PubMed: 2575645]. [PubMed Central: PMC1052869].

14. Akre O, Lipworth L, Cnattingius S, Sparén P, Ekbom A. Risk factor patterns for cryptorchidism and hypospadias. Epidemiology. 1999;10(4):364-9. doi: 10.1097/00001648-199907000-00005.

15. Berkowitz GS, Lapinski RH, Godbold JH, Dolgin SE, Holzman IR. Maternal and neonatal risk factors for cryptorchidism. Epidemiology. 1995;6(2):127-31. doi: 10.1097/00001648-199503000-00007. [PubMed: 7742397].

16. Depue RH. Maternal and gestational factors affecting the risk of cryptorchidism and inguinal hernia. Int J Epidemiol. 1984;13(3):311-8. doi: 10.1093/ije/13.3.311. [PubMed: 6149198].

17. Hutson JM, Hasthorpe S, Heyns CF. Anatomical and functional aspects of testicular descent and cryptorchidism. Endocr Rev. 1997;18(2):25980. doi: 10.1210/edrv.18.2.0298. [PubMed: 9101140].

18. Leveno KJ, Bloom SL, Spong CY, Dashe JS, Hoffman BL, Casey BM Williams obstetrics. 25th ed. United States of America: McGraw-Hill Education; 2020.

19. Calle EE, Khoury MJ. Completeness of the discharge diagnoses as a measure of birth defects recorded in the hospital birth record. Am J Epidemiol. 1991;134(1):69-77. doi: 10.1093/oxfordjournals.aje.a115994. [PubMed: 1853862]. 